\title{
Effect of temperature mismatch on the life cycle of thermoelectric generator efficiency for waste heat recovery
}

\author{
Lim Zi Feng ${ }^{1}$ and Lim Joon Hoong, ${ }^{1, *}$ \\ ${ }^{1}$ School of Computer Science and Engineering, Taylor's University Lakeside Campus, 47500 Subang \\ Jaya, Malaysia
}

\begin{abstract}
Global warming due to greenhouse gases that has been produced by energy generator as a byproduct has becoming a serious issue in recent decades. Thermoelectric module is an alternative method that can generate energy from heat and vice versa. The module is denominated as thermoelectric generator (TEG) when it is used to generate electricity via a process called the Seebeck effect. The use of thermoelectric generator has become more and more demanding due to the low maintenance cost and waste heat availability can be found everywhere in daily life such as car exhaust, roof tiles, and etc. The purpose of this research paper was to determine the effect of temperature mismatch on the life cycle of the thermoelectric generator efficiency using ANSYS simulation. The common used materials for the thermoelectric are bismuth telluride, lead telluride and silicon germanium. Each material has different thermal conductivity, Seebeck coefficient and electrical resistivity. The materials are paired together to form a thermocouple and the thermal gradient of the TEG is being evaluate through the simulation. Generally, the greater the temperature between the hot and cold side of the TEG, the higher the power generated. Bismuth telluride has a highest temperature difference between the hot and cold side followed by lead telluride and silicon germanium. The combination of $\operatorname{BiTe}(\mathrm{N})-\operatorname{BiTe}(\mathrm{P})$ has the lowest minimum heat flux compared to the rest of the thermoelectric material combination. This proves that thermal and electrical properties and combination of thermoelectric material plays a vital role in the thermal gradient of the TEG.
\end{abstract}

\section{Introduction}

Most of the electrical energy in the current society is generated using fossil fuel and nuclear power plants that in general will produce significant amounts of harmful waste such as carbon monoxide and radioactive wastes which causes society problems such as air pollution. Humans are always in a constant search of developing new technologies for renewable energy. Thermoelectric Module (TEM) is a device that can be operated as an electrical generator or as a heating/cooling device. The applications of TEM does not

\footnotetext{
* Corresponding author: JoonHoong.Lim@taylors.edu.my
} 
require any coolants or turbines to work due to its being a solid state device. It is a device that does conversion between electrical and thermal energy. When a TEM is being used as an energy generator, it is denominated as a Thermoelectric Generator (TEG). The amount of electrical energy generated is proportional to the net difference in temperature on each side of the TEG, known as the Seebeck effect. When TEM is being used as a cooler it is denominated as Thermoelectric Cooler (TEC) [1]. When an electric current is being applied to the TEC, it acts as a heat pump which causes the heat to flow from one side of the device to another, known as the Peltier effect. By that, one side of the TEC device will be cooled while the other side will be heated concurrently.

The semiconductor material in the TEM device is the main component and the efficiency of the device is greatly depending on the semiconductor material used. The semiconductor thermocouples in the TEM are in the form of p-type and n-type and they are electrically connected in series and thermally connected in parallel form. The arrays of semiconductor thermocouples are sandwiched in between two ceramic plates. The common material that is commercially used for TEM material is bismuth telluride alloy, $\mathrm{Bi}_{2} \mathrm{Te}_{3}$ [2]. Due to the nature of wear and tear and environmental conditions, the efficiency of the device decreases as the TEM material decreases overtime. Since TEMs do not consist of any mechanical moving parts, it has been suggested that TEGs are reliable power generators when it is being operated under thermo-mechanical stable conditions. However, when a TEM is subjected to thermal cycling, it can undergo severe performance degradation due to the mismatch of thermal expansion coefficient [3]. An induced thermoelectric voltage across each end of the thermoelectric materials will be produced when there is a temperature difference across. The magnitude of the thermoelectric voltage is proportional to the temperature difference across the material. In general cases, the higher the Seebeck coefficient of the material, the higher the efficiency of the material in moving electrons [4,5].

The desirability of the semiconductor material is generally determined by the figure of merit, Z. Figure of merit is usually represented in its dimensionless form, ZT [6]. In general, the higher the figure of merit, the higher the thermal efficiency of the TEG. A higher thermal conductivity of a material will negatively affect the performance of the TEG. The purpose of this project is to conduct an ANSYS workbench simulation of the TEG for to obtain the thermal gradient and voltage generation for different type of material use for the thermoelectric semiconductor [7]. Therefore, determining the parameters that affecting the thermal performance of the TEG is crucial in this research. Furthermore, the thermal and electrical properties of the semiconductor materials are significant in evaluating the performance [8]. Thus, obtaining the correct value parameters is very important. Besides, it is also important to obtain the correct dimension of a TEG and the correct material used. After obtaining all the necessary information, the TEG can now be modelled in Solidworks

\section{Research Methodology}

The modelling of the thermoelectric generator is based on a model SP1848 [9], which is a TEG that is widely available on the market. The TEG is modelled with the exact dimension in Solidworks as shown in Table 1. A full size thermoelectric and a thermocouple is drawn. Due to the complexity of a full size TEG that will consume a lot of computer power when doing simulation, a thermocouple model is used to do setup and Grid Independence Test (GIT) and the data is then transfer to the full size TEG model for extension simulation. A modelled TEG according to the given structural dimension is shown in Figure 1. 
Table 1. Structural dimension of TEG Model SP1848 [9].

\begin{tabular}{|l|c|}
\hline \multicolumn{1}{|c|}{ Description } & Style \\
\hline Leg depth & 1.0 \\
\hline Leg width & 1.0 \\
\hline Leg height & 1.5 \\
\hline Distance between legs & 0.5 \\
\hline Copper link thickness & 0.1 \\
\hline Alumina thickness & 0.2 \\
\hline
\end{tabular}

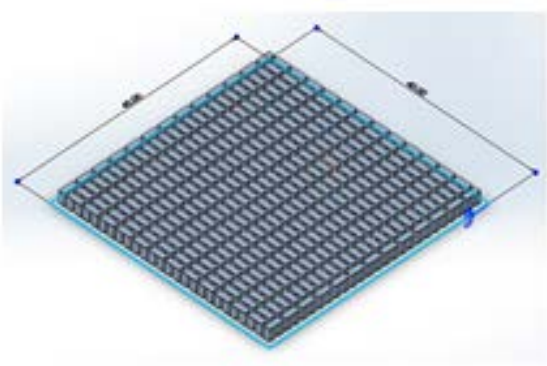

(a)

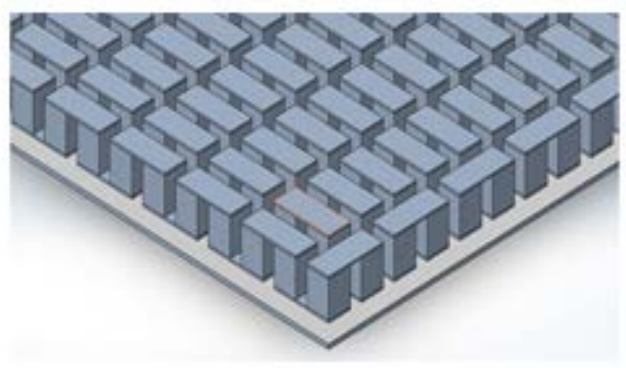

(b)

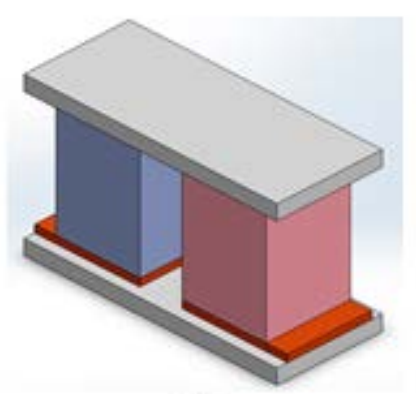

(c)

Fig. 1. (a) Cross section of a full size TEG (b) Close up of an array of thermocouples (c) Single unit of thermocouple.

The FEA model were then inputed with the appropriate properties listed in table one in the setup of ANSYS workbench. Each components were assigned with the specific material accordingly. The model then acquired the most suitable meshing to minimise error in the simulation process. Grid Independent Test was conducted on the model to find the appropriate body sizing to obtain an optimum result and preserve the computer processing power at the same time. During the setup phase, a temperature of $150^{\circ} \mathrm{C}$ is subjected to one side of the TEG. The other side will be left cooling room temperature which is $22^{\circ} \mathrm{C}$. The thermoelements are sandwich in between a copper link and alumina. A voltage of $0 \mathrm{~V}$ is set to the copper link that is attached to the N-type semiconductor and a voltage of $8 \mathrm{mV}$ is set 
to the P-type semiconductor. The thermal and electrical properties of each material are shown in Table 2.

Table 2. Thermal and electrical properties of each material [10-11]

\begin{tabular}{|l|c|c|c|}
\hline \multicolumn{1}{|c|}{ Material } & $\begin{array}{c}\text { Thermal } \\
\text { conductivity } \\
(\mathbf{W} / \mathbf{m K})\end{array}$ & $\begin{array}{c}\text { Seebeck } \\
\text { Coefficient } \\
(\mathbf{V} / \mathbf{K})\end{array}$ & $\begin{array}{c}\text { Electrical } \\
\text { resistivity ( } \mathbf{( \Omega m})\end{array}$ \\
\hline Alumina (Aluminium Oxide) & 35.0 & & \\
\hline Copper & 401.0 & & $1.72 \mathrm{E}-08$ \\
\hline Bismuth Telluride (N-type) & 2.0 & -0.00012 & $1.57 \mathrm{E}-05$ \\
\hline Bismuth Telluride (P-type) & 2.0 & 0.00023 & $2.94 \mathrm{E}-05$ \\
\hline Lead(II) Telluride (PbTe) (N-type) & 3.3 & -0.00015 & $1.00 \mathrm{E}-05$ \\
\hline Lead(II) Telluride (PbTe) (P-type) & 3.3 & 0.00014 & $1.50 \mathrm{E}-05$ \\
\hline $\begin{array}{l}\text { Silicon Germanium (Si[1-x]Ge[x]) } \\
\{\mathrm{x}=0.3\} \text { (N-type) }\end{array}$ & 5.2 & -0.00017 & $1.65 \mathrm{E}-05$ \\
\hline $\begin{array}{l}\text { Silicon Germanium (Si[1-x]Ge[x]) } \\
\{\mathrm{x}=0.3\} \text { (P-type) }\end{array}$ & 5.2 & 0.00011 & $9.60 \mathrm{E}-06$ \\
\hline
\end{tabular}

\section{Results and Discussion}

Grid Independent Test is conducted to find the suitable meshing element size that is effective and efficient to solve for the simulation with this particular model. The structure of a TEG model is suitable to apply a square grid meshing as shown in Figure 2.

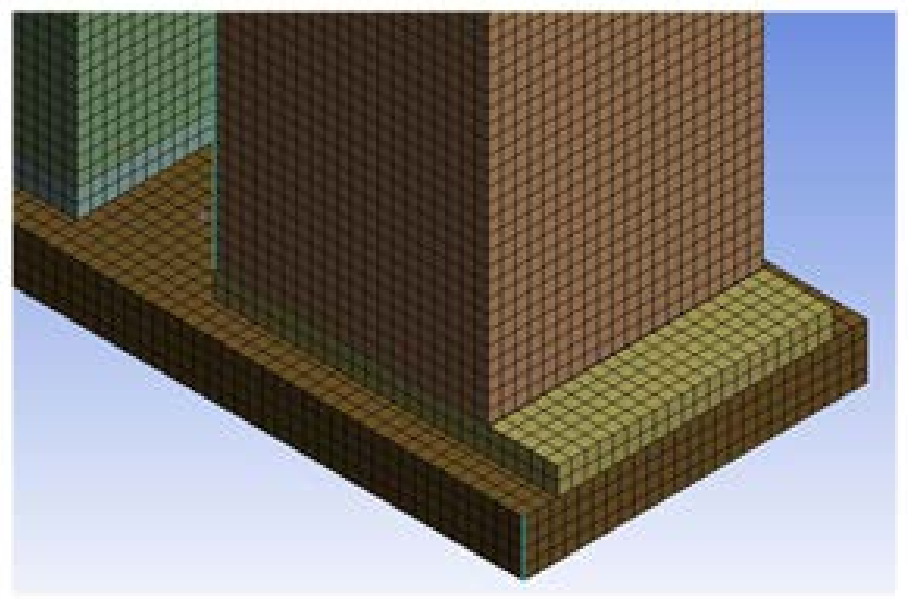

Fig. 2. Square grid meshing of a TEG model

Average temperature is used to determine any significant fluctuations as the mesh size get smaller. The average temperature within the TEG model has no significant difference after mesh size of $0.0001 \mathrm{~m}$ as shown in Figure 3. The mesh size of $0.0001 \mathrm{~m}$ is then used for every simulations with different assigned materials. 


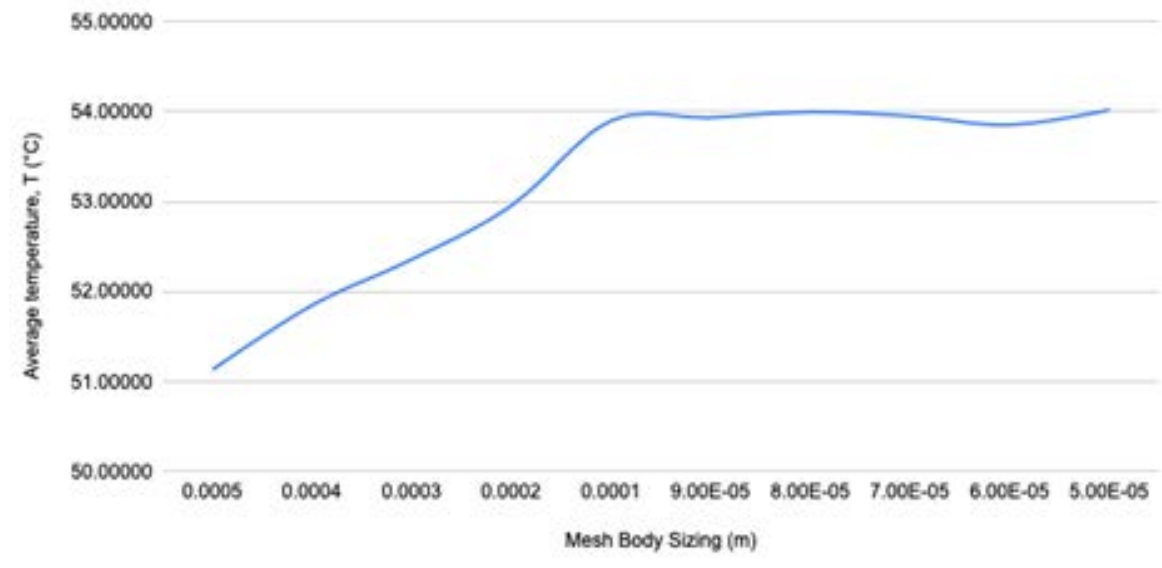

Fig. 3. Grid Independent Test of designed TEG model

The combination of N-junction and P-Junction that is being used for simulation. In this simulation, the data that require to obtain is the average temperature, maximum heat flux and maximum current density across the TEG unit. There are total of 9 combinations of Ntype and P-type semiconductor simulated in this project. Based on the simulated data in Table 3, the combination of semiconductor with the presence of silicon germanium as ptype will generate a higher average temperature within the TEG module. On the other side, the combination with the presence of bismuth telluride as p-type will generate a lower average temperature within the TEG module.

Table 3. Average temperature of different semiconductor combination

\begin{tabular}{|c|c|c|}
\hline N-Junction & P-Junction & $\begin{array}{c}\text { Average } \\
\text { temperature }\left({ }^{\circ} \mathbf{C}\right)\end{array}$ \\
\hline Bismuth Telluride (N-type) & Bismuth Telluride (P-type) & 51.924 \\
\hline Bismuth Telluride (N-type) & Lead(II) Telluride (PbTe) (P-type) & 52.312 \\
\hline Bismuth Telluride (N-type) & $\begin{array}{l}\text { Silicon Germanium }(\operatorname{Si}[1-\mathrm{x}] \mathrm{Ge}[\mathrm{x}]) \\
\{\mathrm{x}=0.3\} \text { (P-type) }\end{array}$ & 52.597 \\
\hline Lead(II) Telluride (PbTe) (N-type) & Bismuth Telluride (P-type) & 51.906 \\
\hline Lead(II) Telluride (PbTe) (N-type) & Lead(II) Telluride (PbTe) (P-type) & 52.169 \\
\hline Lead(II) Telluride (PbTe) (N-type) & $\begin{array}{l}\text { Silicon Germanium }(\operatorname{Si}[1-\mathrm{x}] \mathrm{Ge}[\mathrm{x}]) \\
\{\mathrm{x}=0.3\} \text { (P-type) }\end{array}$ & 52.311 \\
\hline $\begin{array}{l}\text { Silicon Germanium }(\mathrm{Si}[1-\mathrm{x}] \mathrm{Ge}[\mathrm{x}]) \\
\{\mathrm{x}=0.3\}(\mathrm{N} \text {-type) }\end{array}$ & Bismuth Telluride (P-type) & 51.651 \\
\hline $\begin{array}{l}\text { Silicon Germanium }(\operatorname{Si}[1-\mathrm{x}] \mathrm{Ge}[\mathrm{x}]) \\
\{\mathrm{x}=0.3\} \text { (N-type) }\end{array}$ & Lead(II) Telluride (PbTe) (P-type) & 51.737 \\
\hline $\begin{array}{l}\text { Silicon Germanium }(\mathrm{Si}[1-\mathrm{x}] \mathrm{Ge}[\mathrm{x}]) \\
\{\mathrm{x}=0.3\} \text { (N-type) }\end{array}$ & $\begin{array}{l}\text { Silicon Germanium }(\mathrm{Si}[1-\mathrm{x}] \mathrm{Ge}[\mathrm{x}]) \\
\{\mathrm{x}=0.3\} \text { (P-type) }\end{array}$ & 52.362 \\
\hline
\end{tabular}


Based on the simulated data in Figure 4, the maximum current density within the TEG module is the lowest with the presence of bismuth telluride as a p-type. Differently, the maximum current density is the highest within the TEG module with the presence of silicon germanium as the p-type.

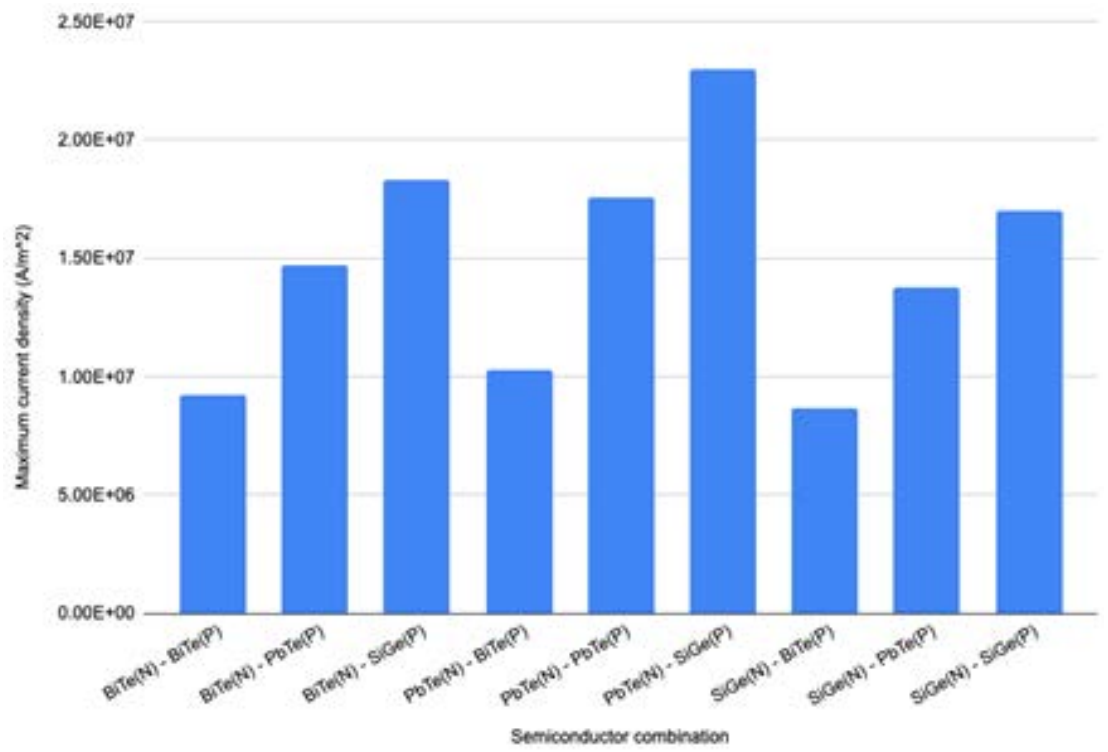

Fig. 4. Grid Independent Test of designed TEG model

Based on the simulated data in Figure 5, semiconductor combination with the presence of silicon germanium regardless of n-type or p-type will generate a marginally higher maximum heat flux within the TEG module compare to other semiconductor material. The combination of $\operatorname{BiTe}(\mathrm{N})$ - $\mathrm{BiTe}(\mathrm{P})$ yield the lowest maximum heat flux among all combination.

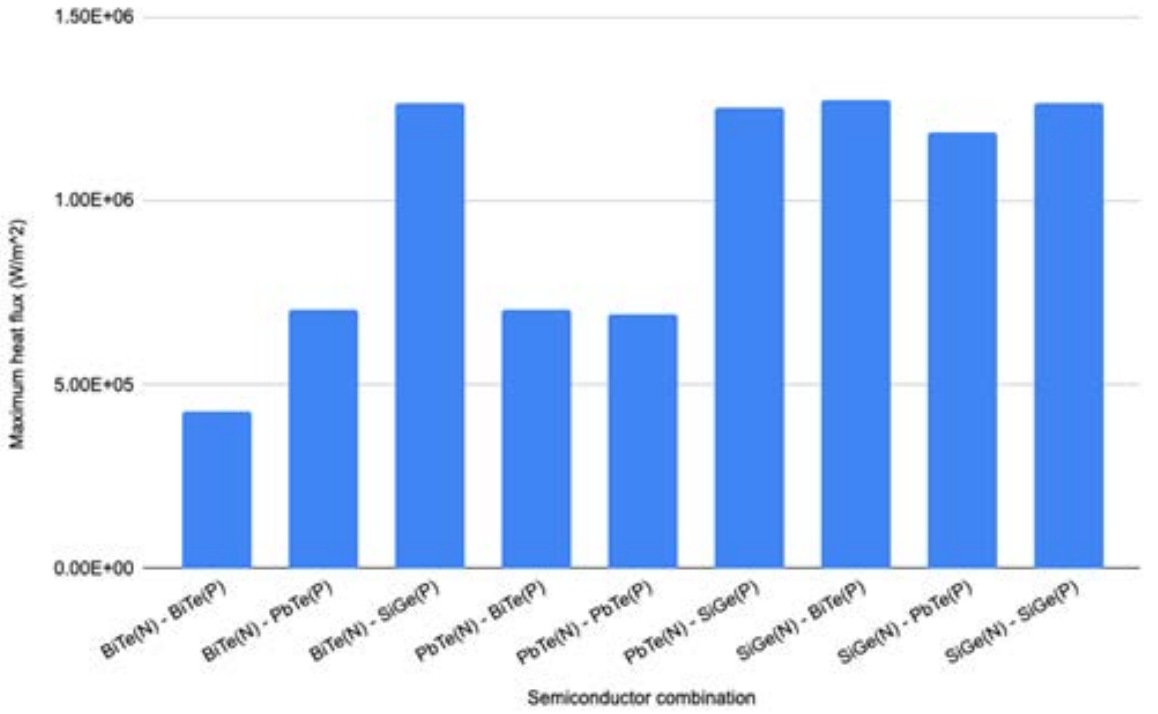

Fig. 5. Grid Independent Test of designed TEG model 


\section{Conclusion}

In recent years, the use of TEG has been increased in many sectors of the society such as mounting it parallel with solar panels. The solar panel gets hotter when in use and the waste heat can be utilised by TEG to again generate electricity in the second run. The gain in popularity of TEG is because it is a source of renewable energy that does not emit any harmful or radioactive substances as product. In this research, the thermal gradient of the TEG is being analysed with the use of computer software such as ANSYS Workbench and Solidworks. Based on the simulated data above, silicon germanium as p-type semiconductor will yield a higher average temperature within the TEG module as compared to other material. Studies suggested that this is due to a higher thermal conductivity of silicon germanium compare to other thermo material in the list. Besides, a p-type silicon germanium has the lowest electrical resistivity among all the thermo material listed. With the use of silicon germanium as a p-type thermo material is able to achieve a higher maximum current density in within the thermocouple as shown in figure 6. Lastly, the ntype and p-type bismuth telluride has a highest electrical resistivity compared to other thermo material. This shows that the combination of $\operatorname{BiTe}(N)-\operatorname{BiTe}(\mathrm{P})$ has the lowest minimum heat flux compared to the rest of the thermo material combination.

\section{References}

1. D. Zhao and G. Tan, "A review of thermoelectric cooling: Materials, modeling and applications", Applied Thermal Engineering, vol. 66, no. 1-2, pp. 15-24, 2014.

2. "Introduction to Thermoelectrics and Medical Applications | Applied Thermoelectric Solutions LLC", Applied Thermoelectric Solutions LLC, 2020.

3. P. Vadivu, P. Sumathy and A. Vadivel, "Ranking images in web documents based on HTML TAGs for image retrieval from WWW", International Journal of Computational Intelligence Studies, vol. 3, no. 23, p. 176, 2014.

4. H. Rocha Liborio Tenorio, D. Vieira and C. De Souza, "Measurement of parameters and degradation of thermoelectric modules", IEEE Instrumentation \& Measurement Magazine, vol. 20, no. 2, pp. 13-19, 2017. Available: 10.1109/mim.2017.7919127.

5. S. Weera, H. Lee and A. Attar, "Utilizing effective material properties to validate the performance of thermoelectric cooler and generator modules", Energy Conversion and Management, vol. 205, p. 112427, 2020. Available:

10.1016/j.enconman.2019.112427.

6. M. Kambe, T. Jinushi and Z. Ishijima, "Encapsulated Thermoelectric Modules and Compliant Pads for Advanced Thermoelectric Systems", Journal of Electronic Materials, vol. 39, no. 9, pp. 1418-1421, 2010.

7. R. Fritts, "Special Applications of Thermoelectric Generators", IEEE Transactions on Industry and General Applications, vol. -3, no. 5, pp. 458-462, 1967.

8. "Measuring semi-conductor properties of thermoelectric materials", Microelectronics Reliability, vol. 13, no. 2, p. 80, 1974. Available: 10.1016/0026-2714(74)90526-5.

9. R. Vrijen et al., "Electron-spin-resonance transistors for quantum computing in silicon-germanium heterostructures", Physical Review A, vol. 62, no. 1, 2000.

10. M. Kambe, T. Jinushi and Z. Ishijima, "Encapsulated Thermoelectric Modules and Compliant Pads for Advanced Thermoelectric Systems", Journal of Electronic Materials, vol. 39, no. 9, pp. 1418-1421, 2010.

11. Merienne, R., Lynn, J., McSweeney, E. and O'Shaughnessy, S., 2019. Thermal cycling of thermoelectric generators: The effect of heating rate. Applied Energy, 237, pp.671681. 